\title{
Comunicação \\ Comunidade de parasitóides associada à cultura do café em Piatã, Chapada Diamantina, BA ${ }^{1}$
}

\author{
Magno Clery da Palma-Santos², Raquel Pérez-Maluf ${ }^{3}$
}

\begin{abstract}
RESUMO
Os himenópteros parasitoides são inimigos naturais de insetos-praga e têm demonstrado eficiência em estratégias de controle, contribuindo para a manutenção do equilíbrio ecológico de agroecossistemas. Esta pesquisa buscou identificar a diversidade de parasitoides associada a culturas de café em Piatã, BA. As coletas foram realizadas com armadilhas Malaise, que permaneceram no campo por sete dias em coletas mensais, de setembro de 2006 a agosto de 2007. Foram coletados 14.669 himenópteros, distribuídos em nove superfamílias, sendo elas Ceraphronoidea, Chalcidoidea, Chrysidoidea, Cynipoidea, Evanioidea, Ichneumonoidea, Mymarommatoidea, Platygastroidea e Proctotrupoidea, e 29 famílias. Coletaram-se 22 famílias constantes e 11 dominantes, destacando-se Ichneumonidae, Braconidae e Scelionidae como mais frequentes, totalizando 50,33\% dos indivíduos coletados. As famílias Braconidae, Eulophidae e Bethylidae, indicadas como promissoras em programas de controle biológico no café, foram coletadas ao longo de todo o ciclo fenológico do café.
\end{abstract}

Palavras-chave: Agricultura orgânica, Coffeea arabica L., inimigos naturais, parasitica.

\section{ABSTRACT}

\section{Parasitoid community associated with coffee crop in Piatã, Chapada Diamantina, Brazil}

Hymenopteran parasitoids are natural enemies of insect pests and have demonstrated efficiency in strategies of biological control, contributing for the maintenance of the ecological balance in agroecosystems. The aim of this research was to identify the diversity of parasitoid wasps in coffee crops in Piatã, BA, Brazil. Malaise traps were installed in the plots and remained in the field for seven days in monthly samplings from September 2006 to August 2007. A total of 14.699 individuals were collected. The parasitoids were distributed through 9 superfamilies: Ceraphronoidea, Chalcidoidea, Chrysidoidea, Cynipoidea, Evanioidea, Ichneumonoidea, Mymarommatoidea, Platygastroidea, Proctotrupoidea and 29 families. Twenty two constant and 11 dominant families, standing out the families Ichneumonidae, Braconidae e Scelionidae as the most frequent. Families Braconidae, Eulophidae and Bethylidae indicated as promising biological control programs in coffee were collected throughout the phenological cycle of the crop.

Key words: Coffea arabica L., natural enemies, organic agroecosystems, parasitica

\footnotetext{
Recebido para publicação em junho de 2008 e aprovado em fevereiro de 2010

${ }^{1}$ Este trabalho é parte da dissertação apresentada ao Programa de Pós-Graduação em Agronomia, Universidade Estadual do Sudoeste da Bahia (UESB)

${ }^{2}$ Biólogo, Mestre em Agronomia. Universidade Estadual do Sudoeste da Bahia, Departamento de Estudos Básicos e Instrumentais, BR 415, Km 03, s/nº, 45700-000, Itapetinga, Bahia, Brasil. clerypiata@gmail.com.

${ }^{3}$ Bióloga, Doutora em Biologie Du Comportement. Universidade Estadual do Sudoeste da Bahia, Laboratório de Biodiversidade do Semi-Árido, Departamento de Ciências Naturais, Estrada do Bem Querer, Km 04 s/n, 45083-900, Vitória da Conquista, Bahia, raquelperezmaluf@gmail.com.
} 


\section{INTRODUÇÃO}

A produção cafeeira na Chapada Diamantina é estimada em 500 mil sacas por ano, tendo na produção de cafés especiais um crescimento significativo, recebendo premiações desde 2003 e conquistando o título de melhor café do Brasil em 2009 pela Associação Brasileira de Cafés Especiais (BSCA) (Alves, 2009). Essa produção é baseada em práticas de manejo que produzem baixo impacto ao meio ambiente, com tendência à redução do uso de pesticidas para o controle de pragas e aumento em mecanismos de controle biológico com a utilização de parasitóides (IBD, 2007).

A relação estreita dos parasitóides com hospedeiros insetos, pragas comuns em culturas agrícolas, constitui uma atividade controladora que minimiza a população de herbívoros, contribuindo para a sustentabilidade do equilíbrio ecológico (Scatolini \& Penteado-Dias, 2003).

Especificamente para algumas pragas-chave da cultura cafeeira, como o bicho-mineiro (Leucoptera coffeella) (Guérin-Mèneville \& Perrottet, 1842), a brocado-café (Hypothenemus hampei) (Ferrari, 1867) e a mosca-das-raízes (Chiromyza vittata) (Wieldman, 1820), já são indicadas espécies de parasitoides das famílias Braconidae, Eulophidae, Bethylidae e Monomachidae (Hymenoptera: Parasitica), que apresentam bom potencial para programas de controle biológico (Cure et al., 1998; Lima et al., 2000; Musetti \& Johnson, 2004; Melo et al., 2007).

Com o intuito de disponibilizar informações sobre a fauna de parasitoides em regiões produtoras de café, o objetivo desta pesquisa foi identificar os parasitoides associados à cultura do café em Piatã, Chapada Diamantina, BA.

\section{MATERIAL E MÉTODOS}

Esta pesquisa foi desenvolvida no município de Piatã, porção Sul da Chapada Diamantina, BA, em três áreas distintas, localizadas na posição geográfica, área $1\left(13^{\circ}\right.$ 13’ 22,3"S; 41 46’ 23,3"W), área 2 (13 13’21,3"S; 41 46' $\left.19,8^{\prime \prime} \mathrm{W}\right)$ e área 3 ( $\left.13^{\circ} 07^{\prime} 03,7^{\prime \prime} \mathrm{S} ; 41^{\circ} 46^{\prime} 43,4^{\prime \prime} \mathrm{W}\right)$. As coletas mensais foram feitas com armadilhas Malaise (Sääksjärvi et al., 2004), instaladas uma em cada área de amostragem, entre setembro de 2006 e agosto de 2007. As armadilhas permaneceram instaladas por sete dias, e os espécimes capturados foram submetidos a uma triagem, identificados em nível de família a partir das chaves propostas por Goulet \& Huber (1993) e conservados em meio líquido (álcool 70\%).

Para a caracterização da comunidade de famílias de parasitoides, foram estimados a riqueza de famílias (S), o índice de diversidade de Shannon (H'), a equitatividade (J), a frequência e a constância, sendo essas famílias clas- sificadas em constantes (W), acessórias (Y) e acidentais (Z) (Silveira Neto et al.,1976) .

\section{RESULTADOS E DISCUSSÃO}

Foram coletados 14.669 parasitoides, distribuídos em nove superfamílias e 29 famílias (Tabela I). Utilizando-se método semelhante em uma área de mata e com cultivo de café convencional em Vitória da Conquista (BA), Santos (2007) coletou oito superfamílias e 23 famílias de parasitoides.

O maior número de famílias coletadas pertence à superfamília Chalcidoidea, equiparando-se a diferentes trabalhos com amostragens de parasitoides em áreas de mata nativa e áreas agrícolas, com uso de armadilhas Malaise (Dall'Oglio et al., 2000; Marchiori \& Penteado-Dias, 2002; Perioto \& Lara, 2003). Tendo em vista as 20 famílias de Chalcidoidea propostas por Goulet \& Huber (1993), 15 foram amostradas nesta pesquisa, indicando a importante diversidade dessa superfamília nos locais de coleta.

Entre os parasitoides coletados, foram encontrados representantes das famílias Agaonidae, Elasmidae, Eucharitidae, Gasteruptidae, Monomachidae e Perilampidae, que foram pouco frequentes em outras amostragens de parasitoides (Azevedo \& Santos, 2000), cujo registro na Bahia ainda não havia sido feito, o que também ocorreu com a família Mymarommatidae, que foi registrada em São Paulo, por Penteado-Dias (2002), e no Espírito Santo e Tocantins, por Bragança et al. (2004).

Verificou-se a ocorrência de 22 famílias de parasitóides constantes, indicando a presença de muitas famílias com presença em mais de $50 \%$ das coletas e 11 dominantes (Tabela 1). Essas famílias foram coletadas em quase todos os meses de amostragem, e com raras exceções houve ausência em um mês ou dois de coleta, evidenciando possível atuação sobre as pragas pelas famílias indicadas para o controle biológico em café (Figura 1).

As famílias mais frequentes foram Ichneumonidae e Braconidae (Tabela 1). Elas estão amplamente distribuídas pelo mundo com 40.000, 60.000 e 3.000 espécies, respectivamente (Wahl, 1993; Sharkey, 1993). Tais famílias exploram diversos hospedeiros, tendo em comum as ordens Lepidoptera, Neuroptera, Diptera e Hemiptera.

Foram encontrados os valores dos índices de diversidade de Shannon $(2,59)$ e eqüitatividade $(0,77)$. Com método semelhante, Santos (2007) obteve os valores de Shannon $(1,96)$ e a equitatividade $(0,64)$. Os trabalhos de Dall’Oglio et al. (2003), Amaral et al. (2005), e Sperber et al. (2004) apresentaram os índices de diversidade de famílias de 2,15, 1,93 e 2,69, respectivamente. Para as últimas 


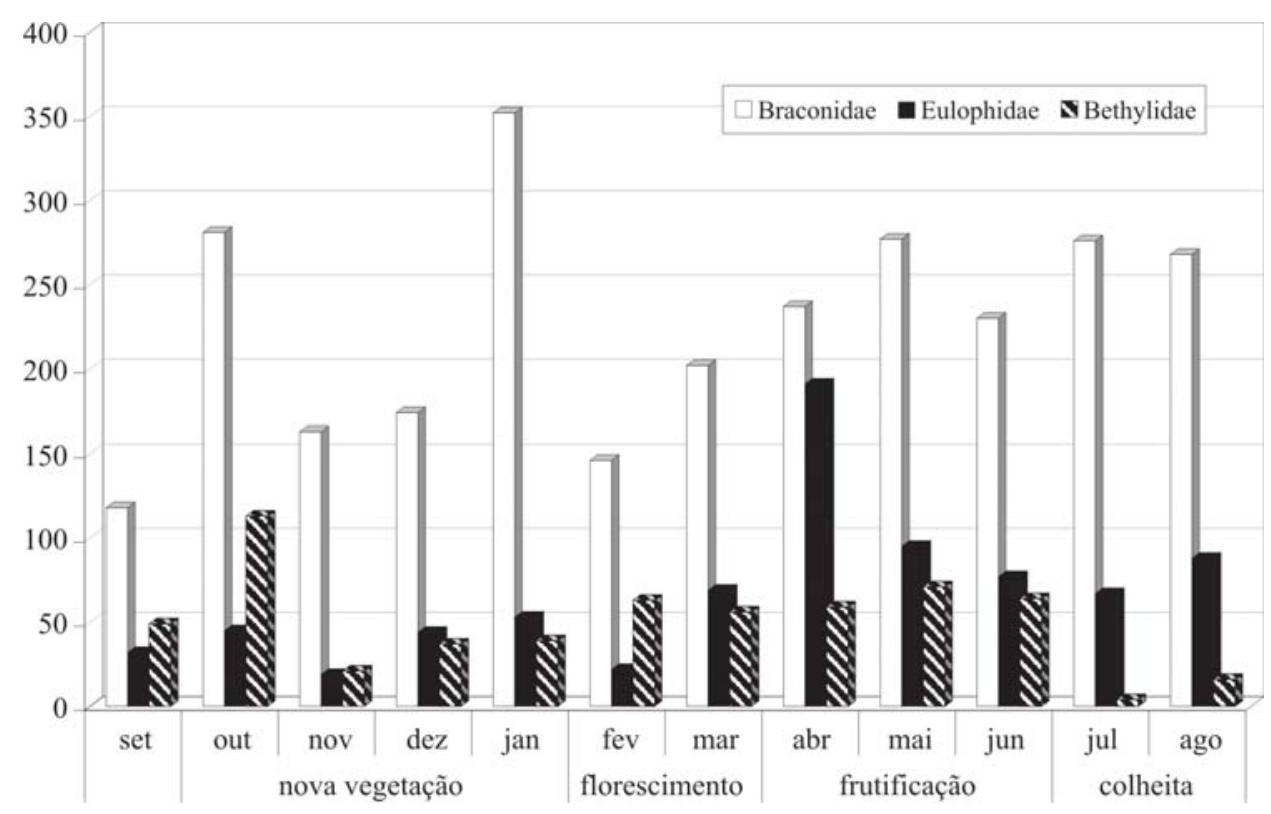

Figura 1. Flutuação das famílias Braconidae, Eulophidae e Bethylidae potenciais em controle de pragas do café, em função do estádio fenológico da cultura.

Tabela 1. Total de himenópteros parasitoides coletados em Piatã, BA, no período de setembro de 2006 a agosto de 2007, Frequência (F), Constância (C: $\mathrm{W}=$ constante (presença nas coletas > de 50\%), Y = acessória (presença nas coletas entre 25 e $50 \%$ ), Z = acidental (presença nas coletas $<25 \%$ ) e Dominância (D: $d$ = dominante $F>1 / S$, nd = não dominante $F<1 / S$ )

\begin{tabular}{lcccc}
\hline Famílias & Total & F & C & D \\
\hline ICHNEUMONOIDEA & 5.759 & - & - & - \\
Ichneumonidae & 3035 & 0,206899 & $\mathrm{~W}$ & $\mathrm{~d}$ \\
Braconidae & 2724 & 0,185698 & $\mathrm{~W}$ & $\mathrm{~d}$ \\
CHALCIDOIDEA & 4.157 & - & - & - \\
Eulophidae & 802 & 0,054673 & $\mathrm{~W}$ & $\mathrm{~d}$ \\
Mymaridae & 774 & 0,052764 & $\mathrm{~W}$ & $\mathrm{~d}$ \\
Pteromalidae & 659 & 0,044925 & $\mathrm{~W}$ & $\mathrm{~d}$ \\
Chalcididae & 507 & 0,034563 & $\mathrm{~W}$ & $\mathrm{~d}$ \\
Encyrtidae & 408 & 0,027814 & $\mathrm{~W}$ & $\mathrm{nd}$ \\
Trichogrammatidae & 372 & 0,02536 & $\mathrm{~W}$ & $\mathrm{nd}$ \\
Aphelinidae & 187 & 0,012748 & $\mathrm{~W}$ & $\mathrm{nd}$ \\
Signiphoridae & 116 & 0,007908 & $\mathrm{~W}$ & $\mathrm{nd}$ \\
Torymidae & 112 & 0,007635 & $\mathrm{~W}$ & $\mathrm{nd}$ \\
Elasmidae & 67 & 0,004567 & $\mathrm{~W}$ & $\mathrm{nd}$ \\
Eupelmidae & 59 & 0,004022 & $\mathrm{~W}$ & $\mathrm{n}$ \\
Perilampidae & 45 & 0,003068 & $\mathrm{Z}$ & $\mathrm{nd}$ \\
Eurytomidae & 37 & 0,002522 & & nd \\
Eucharitidae & 6 & 0,000409 & & \\
\hline
\end{tabular}

pesquisas foram calculados os índices de diversidade a partir dos dados de frequência apresentados nas tabelas.

\section{CONCLUSÕES}

As áreas cafeeiras estudadas são similares quanto à composição das famílias de parasitóides. Registra-se na a Bahia a presença das famílias Mymaromatidae, Agaonidae, Elasmidae, Eucharitidae, Gasteruptidae, Monomachidae e Perilampidae.

\section{REFERÊNCIAS}

Alves, J (2009) Café baiano de Piatã é o melhor do Brasil. Disponível em: <http://www.seagri.ba.gov.br/ noticias.asp?qact=view\&exibir=clipping \& notid=19646>. Acessado em: 10 de janeiro de 2010.

Amaral, DP, Fonseca AR, Silva CG, Silva F M \& Alvarenga Júnior A (2005) Diversidade de famílias de parasitóides (Hymenoptera: Insecta) coletados com armadilhas malaise em floresta nativa em Luz, Estado de Minas Gerais, Brasil. Arquivos do Instituto Biológico, 72: 543-545. 
Azevedo CO \& Santos HS (2000) Perfil da fauna de himenópteros parasitóides (Insecta, Hymenoptera) em uma área de Mata Atlântica da Reserva Biológica de Duas Bocas, Cariacica-ES-Brasil. Boletim do Museu de Biologia Mello Leitão, 11-12:117-126.

Bragança MAL, Acácio RS, Ribeiro RS \& Zanuncio JC (2004) Distribuição e abundância de vespas Mymarommatidae em Mata Atlântica do Espírito Santo e no cerrado do Tocantins. Floresta e Ambiente, 11: 70-72.

Cure JR, Santos RHS, Moraes JC Vilela EF \& Gutierrez AP (1998) Fenologia e dinâmica populacional da broca do café Hypothenemus hampei (Ferr.) relacionadas às fases de desenvolvimento do Fruto. Anais da Sociedade Entomológica do Brasil, 27: 325-335.

Dall’oglio OT, Zanuncio, JC, Azevedo CO \& Medeiros AGB (2000) Survey of the Hymenoptera Parasitoids in Eucalyptus grandis and in a native vegetation area in Ipaba, State of Minas Gerais, Brazil. Anais da Sociedade Entomológica do Brasil, 29: 583-588.

Goulet H \& Huber JT (1993). Hyemenoptera of the word: an identification guide to families. Ottawa, Canada Communication Group. 668 p.

Instituto Biodinâmico de Desenvolvimento Rural (IBD). 2007. Diretrizes para o padrão de qualidade orgânico IBD. $14^{\mathrm{a}}$ ed. Botucatu, Associação Brasileira de Agricultura Biodinâmica. 118p.

Marchiori CH \& Penteado-Dias AM (2002) Famílias de parasitóides coletadas em área de mata e pastagens no município de Itumbiara, Estado de Goiás. Acta Scientiarum, 24: 897-899.

Lima MS, Ribeiro AEL, Boaretto MAC, Brandão ALS, Pujol-Luz JR, Penteado-Dias AM, Santos PRP \& Rocha SAA (2000) Ocorrência de parasitóide na mosca da raiz (Chiromyza vitata Wiedemann) (Diptera: Stratiomyidae) no pólo cafeeiro de Vitória da Conquista, BA. In: $26^{\circ}$ Congresso Brasileiro de Pesquisas Cafeeiras, Marília. Resumos, SDR/PROCAFE/PNFC. p. 196-197.

Melo TL, Castellani MA, Nascimento ML, Menezes Junior AO, Pinto Ferreira GF \& Lemos OL (2007). Comunidades de parasitóides de Leucoptera coffeella (Guérin-Mèneville \& Perrottet, 1842) (Lepidoptera: Lyonetiidae) em cafeeiros nas regiões Oeste e Sudoeste da Bahia. Ciência e Agrotecnologia, 31: 966-972.
Musetti L \& Johnson NF (2004) Revision of the New World species of the genus Monomachus Klug (Hymenoptera: Proctotrupoidea, Monomachidae). Canadian Entomologist, 136: 501-552.

Penteado-Dias A.M (2002) First record of Mymarommatidae (Hymenoptera) from Brazil. Revista Brasileira de Zoologia, 19: 629-631.

Perioto NW \& Lara RIR (2003) Himenópteros parasitóides (Insecta: Hymenoptera) da Mata Atlântica. I. Parque Estadual da Serra do Mar, Ubatuba-SP-Brasil. Arquivos do Instituto Biológico, 70: 441-445.

Sääksjärvi I, Haataja S, Neuvonen S, Gauld ID, Jussila R, Salo J \& Burgos AM (2004) High local species richness of parasitic wasps (Hymenoptera: Ichneumonidae; Pimplinae and Rhyssinae) from the lowland rainforests of Peruvian Amazonia. Ecological Entomology, 29: 735-743.

Santos PS (2007) Diversidade de himenópteros parasitóides em áreas de mata-de-cipó e cafezais em Vitória da Conquista-BA. Dissertação de Mestrado. Universidade Estadual do Sudoeste da Bahia, Vitória da Conquista, 72p.

Scatolini D \& Penteado-Dias AM (2003) Análise faunística de Braconidae (Hymenoptera) em três áreas de mata nativa do Estado do Paraná, Brasil. Revista Brasileira de Entomologia, 47: $187-195$.

Sharkey M J (1993) Family Braconidae. In: Goulet H \& Huber JT (Eds.). Hyemenoptera of the word: an identification guide to families. Ottawa, Canadá Communication Group. p. 362-395.

Silveira Neto S, Nakano O, Barbin D \& Villa Nova NA (1976) Manual de ecologia dos insetos. São Paulo, Editora Agronômica Ceres. 389p.

Sperber FC, Nakayama K, Valverde MJ \& Neves FS (2004) Tree species richness and density affect parasitoid diversity in cacao agroforetry. Basic and Applied Ecology 5: 241-25.

Wahl DB (1993) Family Ichneumonidae. In: Goulet H \& Huber JT (Eds.). Hymenoptera of the word: an identification guide to families. Ottawa, Canadá Communication Group. p. 395-448. 